\title{
MORPHOSPACE FILLING IN FLEXIBLE CRINOIDS
}

KENDRICK, David C., Dept. of Geology, Bryn Mawr College, 101 N. Merion Ave., Bryn Mawr, PA 19010, U.S.A.

Morphospace analysis based on theoretical parameterization breaks down complex geometries into character sets that capture the breadth of morphological possibility. In contrast, empirical morphospaces based on specimen measurement or characters spotlight only the realized subset of potential morphology. In either case, realized forms can be mapped into the resulting morphospaces and explanations generated for differential occupation: biological inviability, developmental canalization, taxonomic preoccupation, chance, etc. Furthermore, both approaches allow large-scale disparity patterns to be inferred from changing patterns of occupation. These different modes of morphospace development are likely to alter the magnitudes and possibly the kinds of patterns we uncover. For example, what appear as discontinuous distributions in an empirical morphospace might instead, in a theoretical space based on growth, be seen as continuous, complicating disparity studies.

In a pilot study, I'm using both paradigms to investigate the range of flexible crinoid morphology through time and the differences in the patterns generated by the two approaches. In the first approach, I model flexible calyxes based on two simple plate parameters and use these parameters to define a morphospace. In the second, I use ordination techniques to define a space based on three dimensional coordinate data from the fossils themselves. Comparing the patterns obtained by mapping the flexibles into both theoretical and empirical spaces suggests that the disparity patterns produced are commensurate, but that theoretical spaces capture the ebb and flow of morphological variation more precisely. 